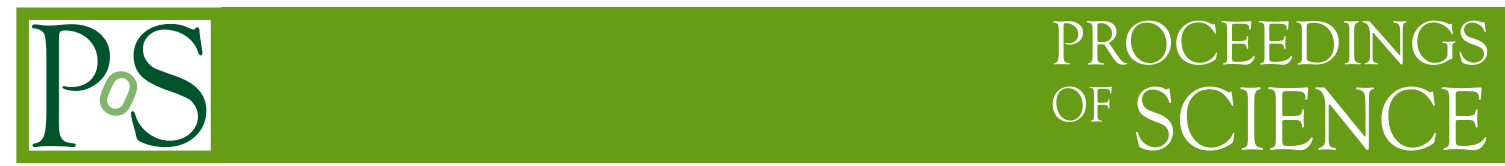

\title{
Searches for dark matter with CMS
}

\section{Isabelle De Bruyn*†}

University of Wisconsin - Madison

E-mail: isabelle.helena.j.de.bruyndcern.ch

Searches for dark matter in many final states with invisible particles recoiling against standard model particles are performed. Various topologies and kinematic variables are explored, as well as jet substructure as a means of tagging heavy bosons. The focus of the talk is on two of the recent CMS results obtained using data collected in the LHC run 2.

European Physical Society Conference on High Energy Physics - EPS-HEP2019 -

10-17 July, 2019

Ghent, Belgium

* Speaker.

${ }^{\dagger}$ on behalf of the CMS Collaboration 


\section{Introduction}

Many astrophysical observations based on the gravitational effects [1] point to the existence of dark matter, however its precise nature remains unknown so far. Innumerable theoretical models are being formulated to explain its origin, and in parallel it is being looked for in numerous experiments. These searches can be categorized in 3 different types, as shown in Fig. 1.

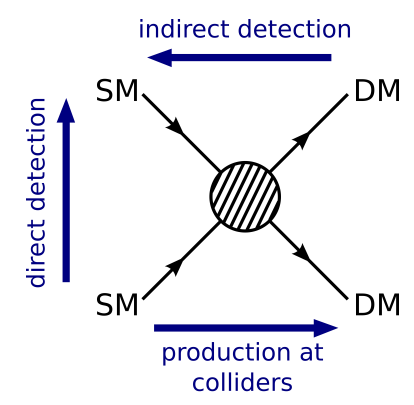

Figure 1: Diagram illustrating the three different methods used to detect dark matter.

The direct detection method is based on the scattering of dark matter particles with nuclei. The indirect detection is aimed at detecting the radiation or particles produced in the annihilation of dark matter particles. Finally, the collider searches try to detect dark matter particles and mediators that could be produced at colliders. The latter category is complementary to direct and indirect searches and includes many different signatures to investigate. This paper focuses on searches at the Compact Muon Solenoid (CMS) experiment [2] located at one of the interaction points of the Large Hadron Collider (LHC) at CERN.

\section{Dark Matter Searches at CMS}

Since dark matter particles are usually assumed to be neutral and to interact only weakly with ordinary matter, they are expected to pass through the detectors at colliders without leaving a signal. These particles can still be searched for when they are produced in association with other visible particles, as this creates an imbalance of transverse momentum. This type of signature is often called the mono- $\mathrm{X}$ signature.

Other types of signatures can be studied as well, when looking for new resonances or longlived particles. However, these searches go beyond the scope of this paper. In this paper, 2 of the recent results based on a mono-X signature are covered: a search for dark matter in association with single top or top quark pairs [3] and the mono-Higgs search [4].

\section{Search for dark matter in association with single top or top quark pairs}

Out of the many possible dark matter interactions, the ones mediated by a new neutral scalar or pseudoscalar particle which then decays to the dark matter particles are especially interesting since they can easily be fit into dark matter models containing extended Higgs boson sectors $[5,6,7,8]$. If the principle of minimal flavor violation $[9,10]$ is respected, the new spin- 0 mediator will follow the Yukawa coupling structure, as is the case in the Standard Model and will favor couplings to the heavy third-generation quarks. 


\subsection{Signature}

When the third-generation quark is a top quark, this leads to the production of dark matter in association with a pair of top quarks ( $\mathrm{t}+\mathrm{t}+\mathrm{DM})$, as detailed in several theoretical studies $[11,12,13$, 14]. The main diagram for this process is shown in Fig. 2 (upper left). The created dark matter escapes detection, leaving an imbalance of momentum in the transverse plane, denoted as $p_{T}^{\text {miss }}$. Additionally, there are contributions from dark matter production in association with a single top quark $(t / \bar{t}+D M)$, as described in Ref. [15]. In this search, these processes which are also mediated by a neutral spin- 0 particle are considered as well for the first time. The relevant diagrams are shown in Fig. 2 (upper right and bottom). Previously, searches for the $t \bar{t}+\mathrm{DM}$ final state have been carried out by the ATLAS and CMS Collaborations at center-of-mass energies of $8 \mathrm{TeV}$ [16, 17] and $13 \mathrm{TeV}[18,19,20]$, based on an effective field theory for the former results and interpreted in the context of simplified models for the latter.
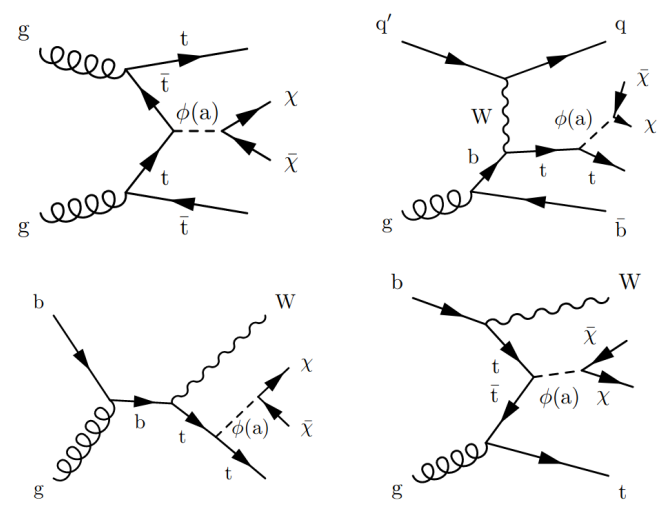

Figure 2: Main production diagrams at the LHC for dark matter in association with a top quark pair (upper left) or a single top quark with associated $t$ channel $\mathrm{W}$ boson production (upper right) or with associated $\mathrm{tW}$ production (lower left and right).

The $\mathrm{t} / \overline{\mathrm{t}}+\mathrm{DM}$ final state is also being studied in the so-called "monotop" searches [21, 22, 23], which involve the production of a top quark and dark matter particles but without the additional jets or W bosons, and considering different simplified models.

\subsection{Analysis method}

In this analysis, the event categorized based on the number of leptons, b-jets, and forward jets. In order to reduce the background in the single lepton signal regions, mainly coming from top quark pairs and $\mathrm{W}$ or $\mathrm{Z}$ bosons produced in association with jets, a cut is imposed on the transverse mass $m_{\mathrm{T}}=\sqrt{2 p_{T}^{m i s s} p_{T}^{l}[1-\cos (\Delta \phi)]}$, where $p_{T}^{l}$ is the transverse momentum of the lepton and $\Delta \phi$ is the opening angle between the lepton direction and the $p_{T}^{\text {miss }}$ vector in the transverse plane.

The different orthogonal signal regions are statistically combined in a simultaneous global fit of the $p_{T}^{\text {miss }}$ spectrum, shown in Fig. 3 for 2 of the signal regions.

\subsection{Results}

The data are in agreement with the expected Standard Model background in the signal regions and the results are interpreted in terms of the described dark matter model with Dirac dark matter. 

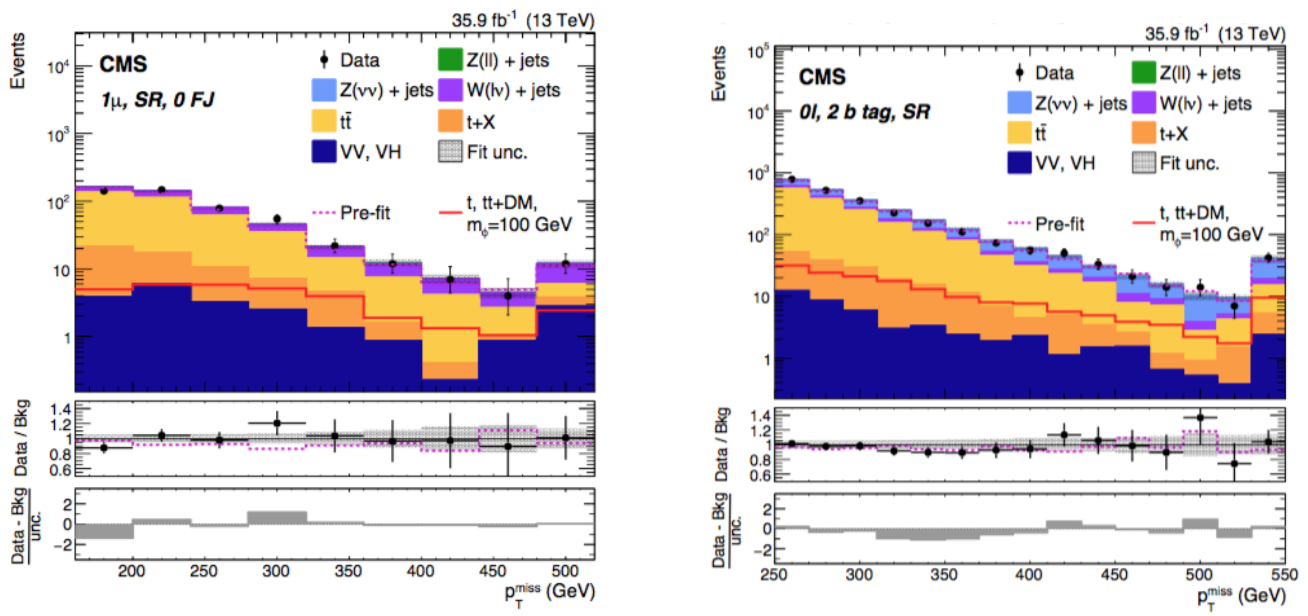

Figure 3: The main observable of the $\mathrm{t} / \overline{\mathrm{t}}+\mathrm{DM}$ search, $p_{T}^{\text {miss }}$, for the signal region with one muon and no forward jets (left), and 2 b-jets and no leptons (right). [3]

The upper limits at 95\% confidence level (CL) are computed on the ratio of the measured and theoretical cross sections, using a modified frequentist approach with a test statistic based on the profile likelihood in the asymptotic approximation and the CLs criterion [24, 25, 26]. The observed and expected limits are shown in Fig. 4 for the scalar and the pseudoscalar models.
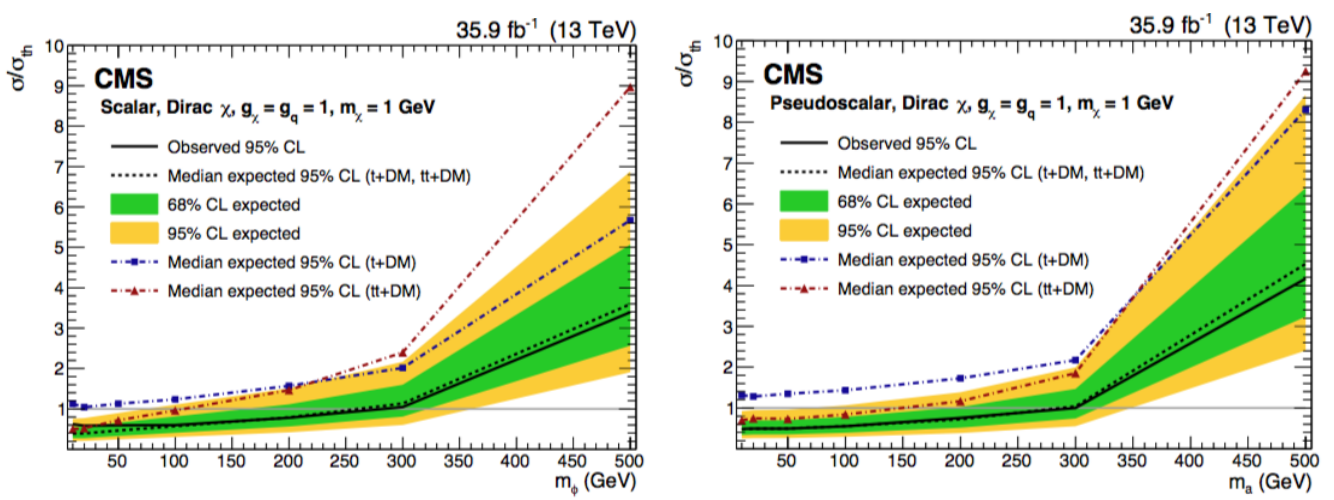

Figure 4: The expected and observed 95\% CL limits on the dark matter production cross sections, relative to the theory predictions, shown for the scalar (left) and pseudoscalar (right) models. [3]

\section{Mono-Higgs search}

With the discovery of the Higgs boson [27, 28, 29] a new probe of dark matter is available, complementing other mono-X searches. The search for pair production of dark matter in association with a Higgs boson differs from typical mono-X searches since the production of the Higgs boson as initial state radiation is strongly suppressed due to the smallness of both the Higgs boson Yukawa coupling to light quarks and its loop-suppressed coupling to gluons. Therefore, the Higgs boson is produced as final state radiation or through a beyond the Standard Model interaction of the dark matter particles with the Higgs boson. 
The mono-Higgs signature has already been studied by both the ATLAS and CMS Collaborations in several Higgs boson decay channels. So far no significant excess of events over the Standard Model expectations has been observed. This paper describes the first search for monoHiggs production which includes the WW and ZZ decay channels, combining this with the results previously obtained in the $b \bar{b}[30,31]$, and $\gamma \gamma$ and $\tau \tau$ [32] channels.

\subsection{Studied models}

Two benchmark simplified models of dark matter production, which are recommended by the ATLAS-CMS Dark Matter Forum [33], are investigated in this search. The corresponding Feynman diagrams are shown in Fig. 5, with a type-II two Higgs doublet model (2HDM) [34] on the left and the baryonic Z' model[35] on the right.
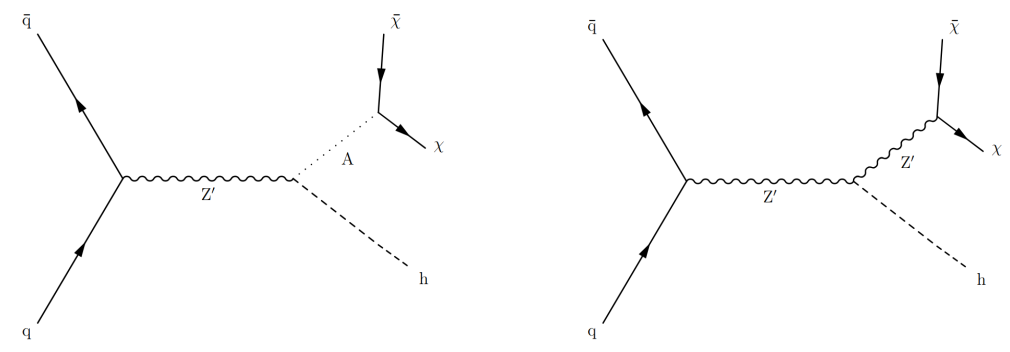

Figure 5: Representative Feynman diagrams for the Z'-2HDM (left) and baryonic Z' (right) models which are considered in the mono-Higgs search.

The $2 \mathrm{HDM}$ model is extended by a $U(1)_{Z^{\prime}}$ group. This model is referred to as the Z'-2HDM model, where the Z' boson is produced via a quark-antiquark interaction, and decays into a Higgs boson and a pseudoscalar A mediator. This mediator can in turn decay into a pair of Dirac fermion DM particles $\chi$.

In the baryonic Z' model, the $Z^{\prime}$ is a vector boson corresponding to a new baryon number $U(1)_{B}$ symmetry, acting as a dark matter mediator. It can radiate a Higgs boson before decaying to a pair of dark matter particles, resulting in the considered signature.

\subsection{Analysis method}

The most sensitive channel for most $Z^{\prime}$ masses is the $b \bar{b}$ channel. In this channel, two separate analyses are performed for the two different signal models. For the Z'-2HDM model the b-jets are expected to be very boosted, resulting into one large-radius jet with 1 or 2 b-tagged subjets. In the baryonic Z' model, the b-jets are expected to be less boosted and the selected signature consists of 2 large-radius jets.

The $\gamma \gamma$ and $\tau \tau$ decay channels have a smaller branching fraction but the former allows for a higher precision in reconstructed invariant mass while the latter has a smaller background due to the leptons in the final state. For the $\gamma \gamma$ result, a fit is performed in the diphoton invariant mass to extract the signal yield. In the $\tau \tau$ case, the signal is extracted using a simultaneous fit to the transverse mass of missing energy and the $2 \tau$ leptons in signal and control regions.

In the WW channel, the kinematic reconstruction of the Higgs boson is not possible due to the neutrinos present in the final state. A BDT is used, trained separately for each signal model. 
The backgrounds in the $\mathrm{ZZ}$ channel are easily reducible and the mass of the Higgs can be fully reconstructed. However, the branching fraction is very small.

\subsection{Results}

The obtained observed and expected 95\% CL upper limits on the cross section are given in Fig. 6 for the separate decay channels and for the combined result. The interpretation of the results in the baryonic Z' model in terms of the spin-independent dark matter nucleonscattering cross section yields higher sensitivity than the existing results from direct detection experiments for dark matter particle masses less than $5 \mathrm{GeV}$, for the chosen benchmark model parameters.
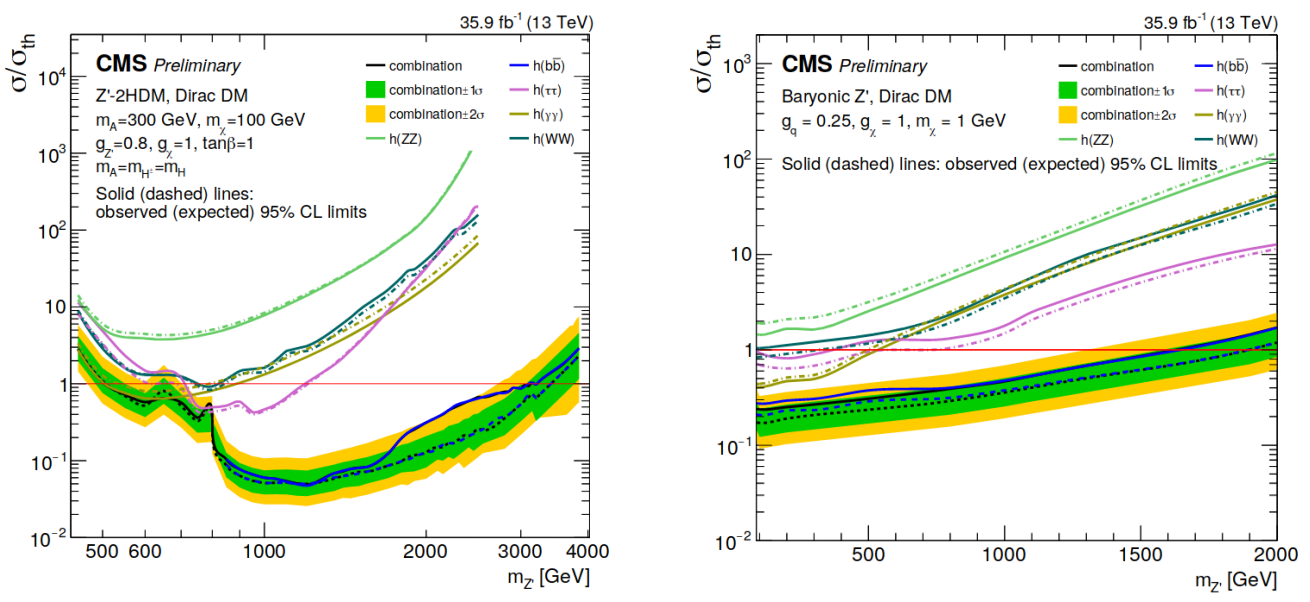

Figure 6: The observed and expected 95\% CL upper limits on $\sigma / \sigma_{t h}$ for the Z'-2HDM (left) and baryonic Z'(right) models for the five individual decay modes of the Higgs boson, as well as their combination. Various mass hypotheses for the $Z^{\prime}$ are considered for a fixed value of $m_{A}=300 \mathrm{GeV}\left(m_{\chi}=1 \mathrm{GeV}\right)$ for the Z'-2HDM (baryonic Z') model. [4]

\section{Summary}

Many searches for dark matter are being performed, including searches at colliders. In this paper we discussed the results and considered models of two of the searches performed at the CMS experiment. One is the first search at the LHC for dark matter in association with a single top or top quark pair in scalar and pseudoscalar interactions, obtaining an improvement of up to two times on the set limits at high mediator masses with respect to the precious $\bar{t} \bar{t}+\mathrm{DM}$ results. For the mono-Higgs analysis, the main decay channel is the $\mathrm{h} \rightarrow \mathrm{b} \overline{\mathrm{b}}$ channel, while the $\gamma \gamma$ and $\tau \tau$ channels contribute at low Z' mass. This combined analysis for the first time includes the Higgs boson decay channel to two $\mathrm{W}$ or $\mathrm{Z}$ bosons.

\section{References}

[1] G. Bertone, D. Hooper and J. Silk, "Particle dark matter: Evidence, candidates and constraints", Phys. Rept. 405 (2005) 279, doi:10.1016/j.physrep.2004.08.031, arXiv:hep-ph/0404175

[2] CMS Collaboration, "The CMS experiment at the CERN LHC”, JINST 3:S08004,2008 
[3] CMS Collaboration, "Search for dark matter produced in association with a single top quark or a top quark pair in proton-proton collisions at $\sqrt{s}=13 \mathrm{TeV}$ ”, JHEP 1903 (2019) 141, doi:10.1007/JHEP03(2019)141, arXiv:1901.01553

[4] CMS Collaboration, "Search for dark matter particles produced in association with the Higgs boson in proton-proton collisions at $\sqrt{s}=13 \mathrm{TeV}$ ", CMS-PAS-EXO-18-011, http://cds.cern.ch/record/2665233

[5] Y. G. Kim, K. Y. Lee, C. B. Park and S. Shin, "Secluded singlet fermionic dark matter driven by the Fermi gamma-ray excess”, Phys. Rev. D 93 (2016) no.7, 075023, doi:10.1103/PhysRevD.93.075023, arXiv:1601.05089

[6] A. Berlin, S. Gori, T. Lin and L. T. Wang, "Pseudoscalar Portal Dark Matter", Phys. Rev. D 92 (2015) 015005, doi:10.1103/PhysRevD.92.015005, arXiv:1502.06000

[7] Y. G. Kim, K. Y. Lee and S. Shin, “Singlet fermionic dark matter”, JHEP 0805 (2008) 100, doi:10.1088/1126-6708/2008/05/100, arXiv:0803.2932

[8] L. Lopez-Honorez, T. Schwetz and J. Zupan, "Higgs portal, fermionic dark matter, and a Standard Model like Higgs at 125 GeV”, Phys. Lett. B 716 (2012) 179, doi:10.1016/j.physletb.2012.07.017, arXiv:1203.2064

[9] G. D’Ambrosio, G. F. Giudice, G. Isidori and A. Strumia, "Minimal flavor violation: An Effective field theory approach”, Nucl. Phys. B 645 (2002) 155, doi:10.1016/S0550-3213(02)00836-2, arXiv:hep-ph/0207036

[10] G. Isidori and D. M. Straub, "Minimal Flavour Violation and Beyond”, Eur. Phys. J. C 72 (2012) 2103, doi:10.1140/epjc/s10052-012-2103-1, arXiv:1202.0464

[11] T. Lin, E. W. Kolb and L. T. Wang, "Probing dark matter couplings to top and bottom quarks at the LHC”, Phys. Rev. D 88 (2013) no.6, 063510, doi:10.1103/PhysRevD.88.063510, arXiv:1303.6638

[12] M. R. Buckley, D. Feld and D. Goncalves, "Scalar Simplified Models for Dark Matter", Phys. Rev. D 91 (2015) 015017, doi:10.1103/PhysRevD.91.015017, arXiv:1410.6497

[13] U. Haisch and E. Re, "Simplified dark matter top-quark interactions at the LHC", JHEP 1506 (2015) 078, doi:10.1007/JHEP06(2015)078, arXiv:1503.00691

[14] U. Haisch, F. Kahlhoefer and J. Unwin, "The impact of heavy-quark loops on LHC dark matter searches”, JHEP 1307 (2013) 125, doi:10.1007/JHEP07(2013)125, arXiv:1208.4605

[15] D. Pinna, A. Zucchetta, M. R. Buckley and F. Canelli, "Single top quarks and dark matter", Phys. Rev. D 96 (2017) no.3, 035031, doi:10.1103/PhysRevD.96.035031, arXiv:1701.05195

[16] CMS Collaboration, "Search for the production of dark matter in association with top-quark pairs in the single-lepton final state in proton-proton collisions at sqrt(s) = $8 \mathrm{TeV}$ ”, JHEP 1506 (2015) 121, doi:10.1007/JHEP06(2015)121, arXiv:1504.03198

[17] ATLAS Collaboration, "Search for dark matter in events with heavy quarks and missing transverse momentum in $p p$ collisions with the ATLAS detector", Eur. Phys. J. C 75 (2015) no.2, 92, doi:10.1140/epjc/s10052-015-3306-z, arXiv:1410.4031

[18] CMS Collaboration, "Search for dark matter produced in association with heavy-flavor quark pairs in proton-proton collisions at $\sqrt{s}=13$ TeV”, Eur. Phys. J. C 77 (2017) no.12, 845, doi:10.1140/epjc/s10052-017-5317-4, arXiv:1706.02581

[19] ATLAS Collaboration, "Search for dark matter produced in association with bottom or top quarks in $\sqrt{s}=13 \mathrm{TeV}$ pp collisions with the ATLAS detector", Eur. Phys. J. C 78 (2018) no.1, 18, doi:10.1140/epjc/s10052-017-5486-1, arXiv:1710.11412 
[20] CMS Collaboration, "Search for dark matter particles produced in association with a top quark pair at $\sqrt{s}=13$ TeV”, Phys. Rev. Lett. 122 (2019) no.1, 011803, doi:10.1103/PhysRevLett.122.011803, arXiv:1807.06522

[21] ATLAS Collaboration, "Search for invisible particles produced in association with single-top-quarks in proton-proton collisions at $\sqrt{s}=8 \mathrm{TeV}$ with the ATLAS detector", Eur. Phys. J. C 75 (2015) no.2, 79, doi:10.1140/epjc/s10052-014-3233-4, arXiv:1410.5404

[22] CMS Collaboration, "Search for Monotop Signatures in Proton-Proton Collisions at $\sqrt{s}=8$ TeV", Phys. Rev. Lett. 114 (2015) no.10, 101801, doi:10.1103/PhysRevLett.114.101801, arXiv:1410.1149

[23] CMS Collaboration, "Search for dark matter in events with energetic, hadronically decaying top quarks and missing transverse momentum at $\sqrt{s}=13$ TeV”, JHEP 1806 (2018) 027, doi:10.1007/JHEP06(2018)027, arXiv:1801.08427

[24] T. Junk, "Confidence level computation for combining searches with small statistics", Nucl. Instrum. Meth. A 434 (1999) 435, doi:10.1016/S0168-9002(99)00498-2, arXiv:hep-ex/9902006

[25] A. L. Read, "Presentation of search results: The CL(s) technique", J. Phys. G 28 (2002) 2693, doi:10.1088/0954-3899/28/10/313

[26] G. Cowan, K. Cranmer, E. Gross and O. Vitells, "Asymptotic formulae for likelihood-based tests of new physics”, Eur. Phys. J. C 71 (2011) 1554, Erratum: [Eur. Phys. J. C 73 (2013) 2501], doi:10.1140/epjc/s10052-011-1554-0, 10.1140/epjc/s10052-013-2501-z, arXiv:1007.1727

[27] ATLAS Collaboration, "Observation of a new particle in the search for the Standard Model Higgs boson with the ATLAS detector at the LHC", Phys. Lett. B 716 (2012) 1, doi:10.1016/j.physletb.2012.08.020, arXiv:1207.7214

[28] CMS Collaboration, "Observation of a New Boson at a Mass of $125 \mathrm{GeV}$ with the CMS Experiment at the LHC”, Phys. Lett. B 716 (2012) 30, doi:10.1016/j.physletb.2012.08.021, arXiv:1207.7235

[29] CMS Collaboration, "Observation of a New Boson with Mass Near $125 \mathrm{GeV}$ in $p p$ Collisions at $\sqrt{s}=$ 7 and 8 TeV”, JHEP 1306 (2013) 081, doi:10.1007/JHEP06(2013)081, arXiv:1303.4571

[30] CMS Collaboration, "Search for heavy resonances decaying into a vector boson and a Higgs boson in final states with charged leptons, neutrinos and b quarks at $\sqrt{s}=13 \mathrm{TeV}$ ", JHEP 1811 (2018) 172, doi:10.1007/JHEP11(2018)172, arXiv:1807.02826

[31] CMS Collaboration, "Search for dark matter produced in association with a Higgs boson decaying to a pair of bottom quarks in protonâĂŞproton collisions at $\sqrt{s}=13 \mathrm{TeV}$ ”, Eur. Phys. J. C 79 (2019) no.3, 280, doi:10.1140/epjc/s10052-019-6730-7, arXiv:1811.06562

[32] CMS Collaboration, "Search for dark matter produced in association with a Higgs boson decaying to $\gamma \gamma$ or $\tau^{+} \tau^{-}$at $\sqrt{s}=13 \mathrm{TeV}$ ", JHEP 1809 (2018) 046, doi:10.1007/JHEP09(2018)046, arXiv:1806.04771

[33] D. Abercrombie et al., "Dark Matter Benchmark Models for Early LHC Run-2 Searches: Report of the ATLAS/CMS Dark Matter Forum”, doi:10.1016/j.dark.2019.100371, arXiv:1507.00966

[34] G. C. Branco, P. M. Ferreira, L. Lavoura, M. N. Rebelo, M. Sher and J. P. Silva, "Theory and phenomenology of two-Higgs-doublet models", Phys. Rept. 516 (2012) 1, doi:10.1016/j.physrep.2012.02.002, arXiv:1106.0034

[35] L. Carpenter, A. DiFranzo, M. Mulhearn, C. Shimmin, S. Tulin and D. Whiteson, "Mono-Higgs-boson: A new collider probe of dark matter", Phys. Rev. D 89 (2014) no.7, 075017, doi:10.1103/PhysRevD.89.075017, arXiv:1312.2592 